\title{
Some corrections to the data analysis of the dynamic tensile tests in the Hopkinson bar
}

\author{
J. Rodríguez, C. Navarro* and V. Sánchez-Gálvez \\ Department of Materials Science, Polytechnic University of Madrid, E.T.SI. de Caminos, Canales y \\ Puertos, Ciudad Universitaria s/n, 28040 Madrid, Spain \\ * Department of Engineering, Carlos III University, Avda. de Butarque 15, Leganés, 28911 Madrid, Spain
}

\begin{abstract}
Résumé. On a fait une étude experimentale et numérique des essais de traction à la barre Hopkinson pour assurer la validité de cette technique pour la caractérisation des matériaux à traction à vitesses de contraintes autour de $1000 \mathrm{~s}^{-1}$. Les champs de contraintes et de deformations dans l'éprouvette sont étudiés par moyen de la méthode des éléments finis pour constater les hypothèses propres de l'essai: situation d'équilibre et distribution homogène des deformations dans l'éprouvette. La simulation fournit aussi des critères pour dessiner mieux l'essai et les dimensions de l'éprouvette de sorte qu'on s'approche à la situation idéale. Finalement, à cause de l'absence d'uniformité de la distribution des déformations on propose des corrections à faire pour obtenir des proprietés valables des matériaux à partir des résultats des essais.
\end{abstract}

\begin{abstract}
An experimental and numerical analysis of tensile tests in the Hopkinson bar is made to assess the suitability of this method to characterize materials in tension conditions at strain rates about $1000 \mathrm{~s}^{-1}$. The stress and strain fields that appear in the specimen are analyzed by the finite element method to check the hypotheses normally used in obtaining results: equilibrium situation and uniform strain distribution in the specimen. The simulation process also provides some criteria to design the experimental set up and the specimen geometry for the best agreement with the ideal situation. Finally, due to the non-uniform strain distribution in the specimen, some corrections are suggested to obtain reliable material properties from test results.
\end{abstract}

\section{INTRODUCTION.}

The development of testing methods based on the Hopkinson bar [1] was a significant breakthrough in the mechanical characterization of materials at high strain rates. All dynamic tests in the Hopkinson bar both in tension and in compression, rest on two basic assumptions: firstly the specimen remains always in quasi-static equilibrium, which means that the inertial forces are negligible and only a single value of the stress acting on the specimen can be given; and secondly the specimen is subjected to a uniform strain field. The first assumption may be not fulfilled during the whole test, at least for the first few microseconds, and the results in that non-equilibrium phase are not representative of any material behaviour. Determining the time when the results begin to be representative of the material is a problem in this experimental device. Regarding the second assumption, in practice, the forces acting on the specimen faces are always known because they are obtained directly from the strains measured on the bars, whose material properties have been previously determined. However, the true strain state in the specimen is more difficult to define, particularly if the specimen geometry is not conventional. There is no direct method to compute the specimen strain field without a sophisticated instrumentation of the specimen, and it is assumed as constant through the entire specimen and is usually calculated directly from the relative displacements of the bar faces in contact with the specimen. This paper analyzes the stress and strain fields within the specimen during dynamic tensile tests in the Hopkinson bar device, and determines the reliability of the quoted assumptions. Different experiments using the device, and their corresponding numerical simulations, have been carried out to investigate the main characteristics of the test. 


\section{EXPERIMENTAL PROGRAMME.}

Basically, the experimental set up includes a cylindrical tensile specimen threaded to two highstrength steel bars of $22 \mathrm{~mm}$ diameter and 1 meter length. The experimental system is depicted in figure 1 .

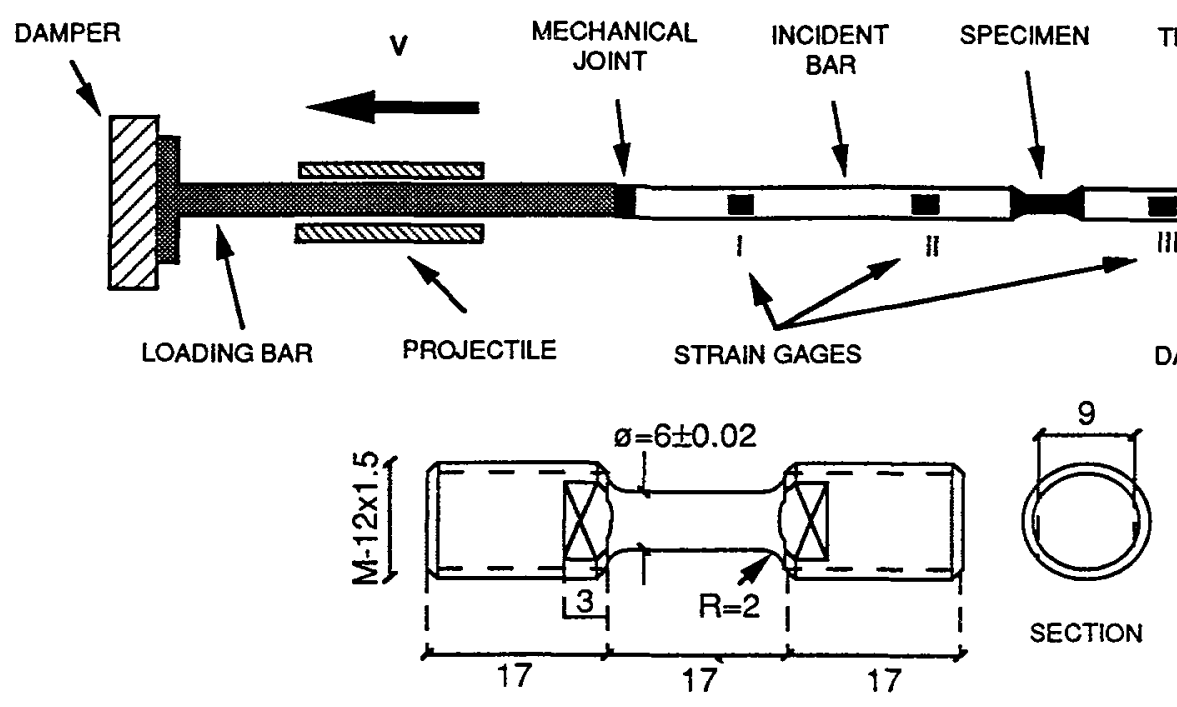

Figure 1. Experimental set up diagram (Dimensions in $\mathrm{mm}$ )

The tensile pulse traveling along the incident bar is partially transmitted to the second bar (transmitter bar) through the specimen. The stress in the specimen is calculated by dividing the transmitted force to the second bar by the specimen section; the strain rate in the specimen is obtained by dividing the relative velocity of the bar ends, which can be obtained from the velocity histories of the incident and transmitting bar edges, by the initial gauge length; and finally, the strain is calculated by integrating the strain rate in time [2].

To compare numerical modeling results with experimental values, a dynamic testing program was carried out. The material used in all tests was 7017 T73 aluminium alloy. This material, like most highstrength aluminium alloys [3], has very little strain rate sensitivity, therefore it is easier to make comparisons between quasi-static and dynamic tests. Quasi-static tests were carried out in a conventional testing machine: the stress in the specimen was determined through a load cell and the strain by means of a $12 \mathrm{~mm}$ gauge extensometer. In both, static and dynamic conditions, specimens had the same geometry (figure 1). The main results are summarized and compared to other ones from the literature [4] in Table 1. Yield stress increases slightly with strain rate, and in fact, the mechanical behaviour of the material tested in this work can be considered as strain rate independent for the strain rate range analyzed. The complete stress-strain curves are shown in figure 2 . To check the validity of the uniform stress distribution hypothesis, tests were carried out on specimens instrumented with strain gauges of $3.18 \mathrm{~mm}$ in length bonded to the specimen shaft. Figure 3 shows the differences between the time-histories of the direct measurement given by the strain gauge placed on the specimen shaft, and that computed from the relative displacement at the bar edges (average strain). As it is seen in figure 3, the material in the specimen was subjected to a non uniform strain distribution. Corrections should be made in this type of test to account for the actual deformation of the material.

Table 1. Comparison of main results.

\begin{tabular}{|l|c|c|}
\cline { 2 - 3 } \multicolumn{1}{c|}{} & Strain Rate $\left(\mathbf{s}^{-1}\right)$ & Yield Stress (MPa) \\
\hline AISI stainless steel & $10^{-4} / 10^{3}$ & $500 / 750$ \\
\hline Al 6061 T651 & $10^{-4} / 10^{3}$ & $300 / 330$ \\
\hline Al 7075 T6 & $10^{-4} / 10^{3}$ & $550 / 700$ \\
\hline Al 7017 T73 $\left(^{*}\right)$ & $10^{-4} / 10^{3}$ & $440 / 500$ \\
\hline
\end{tabular}




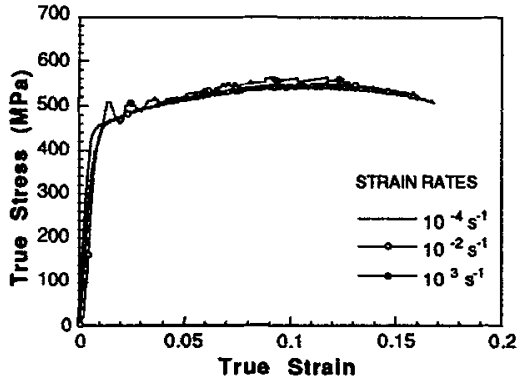

Figure 2. Stress-Strain curves for Al 7017 T73

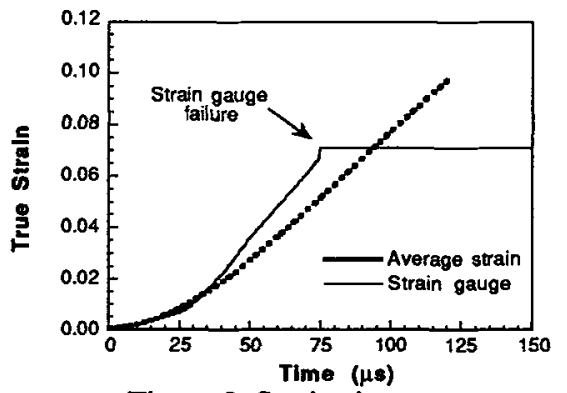

Figure 3. Strain-time curve

\section{NUMERICAL SIMULATION OF THE TESTS.}

To explain the experimental observations and to investigate the actual strain field generated in a specimen in a tension test in the Hopkinson bar device, numerical simulations of the test were carried out using the commercial computer code ABAQUS [5]. The finite element mesh used in computations had 937 nodes and 276 bilinear quadrilateral elements. Due to the axial symmetry of the problem, only the material from the axis to the specimen surface was modeled. The material behaviour was assumed to be bilinear elastic-plastic and strain rate independent with a Young's modulus of $71 \mathrm{GPa}$, Poisson's ratio 0.33 , density $2700 \mathrm{~kg} / \mathrm{m}^{3}$, yield stress $500 \mathrm{MPa}$ and stress-strain slope in the plastic region of about 0.62 $\mathrm{GPa}$. As boundary condition, a trapezoidal velocity pulse was applied to one edge of the specimen. The pulse characteristics were: total duration $220 \mu \mathrm{s}$, rise time $60 \mu \mathrm{s}$, falling time $40 \mu \mathrm{s}$ and maximum value $14 \mathrm{~m} / \mathrm{s}$. This load curve corresponds to that recorded experimentally in the incident bar. To show the appropriateness of the numerical model, a comparison between experimental and numerical values for the force transmitted to the second bar and the strain in the central zone of the specimen has been made, showing an excellent agreement up to specimen necking.

Numerical results can be divided in two parts: quasi-static equilibrium state and uniform strain:

a) Quasi-static equilibrium state: To confirm when the specimen reaches a quasi-static equilibrium, the dynamic stress state at different times was compared with that of a static case (see Figure 4). The stress distribution is symmetrical to the specimen centre, and it is not possible to assess in which of the two specimen edges the load was applied: the specimen is in a static equilibrium state. In a dynamic test, as can be seen in Figure 4, the stress field is not yet symmetrical at 5 and $10 \mu \mathrm{s}$, therefore the force applied on the specimen cannot be stated. However, at $15 \mu \mathrm{s}$, the situation has evolved and the stress state is identical to that obtained from a quasi-static test (see Figure 4). Since that time, the stress in the specimen can be calculated directly from the force measured in the bars. Taking into account, that $60 \mu \mathrm{s}$ is a bit larger rise time than usual, two other numerical analyses have been carried out with shorter rise times: 40 and $20 \mu s$. Numerical analyses show that the time needed to reach the equilibrium state does not vary and the only difference is, obviously, the stress level in the specimen. This result seems logical, because the equilibrium state is usually assumed to be reached after several elastic wave reverberations in the specimen. This last statement is also confirmed by a numerical analysis considering a longer specimen.

b) Uniform strain: The experimental discrepancies between the average strain computed by the traditional method and the strain directly measured in the specimen are also confirmed by the numerical model, in which the actual strain distribution is analyzed in detail at different times, as shown in Figure 5. At $30 \mu \mathrm{s}$ the elastic strain is almost uniform along the gauge length, although the zone near the bars is also deformed. At $60 \mu \mathrm{s}$, in the middle of the plastic flow regime, the strain distribution in the gauge length is not uniform, important differences appearing between strains in the central zone and those nearer the threads. That situation continues during the whole plastic flow until necking starts. 
QUASI-STATIC

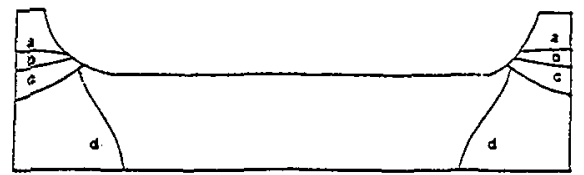

$(a=18 \mathrm{MPa} ; b=40 \mathrm{MPa} ; c=62 \mathrm{MPa} ; d=84 \mathrm{MPa})$

DYNAMIC $(10 \mu s)$

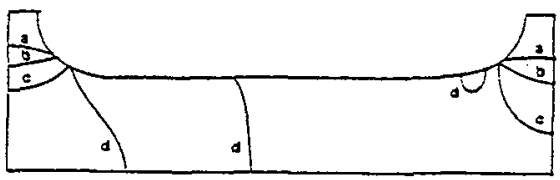

$(a=11 \mathrm{MPa} ; b=24 \mathrm{MPa} ; c=37 \mathrm{MPa} ; d=50 \mathrm{MPa})$
DYNAMIC $(5 \mu \mathrm{s})$

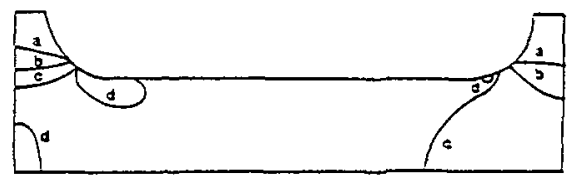

$(a=3 \mathrm{MPa} ; b=7 \mathrm{MPa} ; c=11 \mathrm{MPa} ; d=15 \mathrm{MPa})$

DYNAMIC $(15 \mu s)$

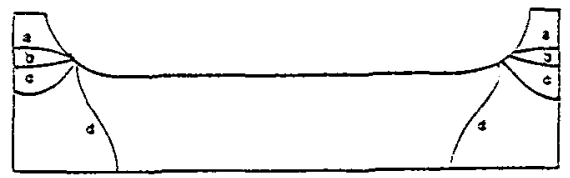

$(a=22 \mathrm{MPa} ; b=48 \mathrm{MPa} ; c=74 \mathrm{MPa} ; d=100 \mathrm{MPa})$

Figure 4. Numerical Stress Contours

ELASTIC REGLEE ( $30 \mu \mathrm{s})$

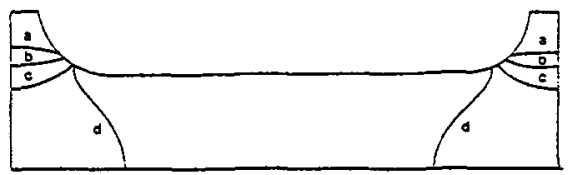

$(a=0.15 \% ; b=0.28 \% ; c=0.42 \% ; d=0.55 \%)$
PLASTIC REGIME (60 $\mu s)$

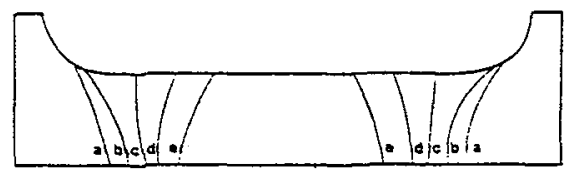

$(a=0.69 \% ; b=1.37 \% ; c=2.05 \% ; d=2.73 \% ; e=3.40 \%)$

Figure 5. Numerical Strain Contours

\section{DISCUSSION.}

In the numerical analyses carried out, the equilibrium situation is clearly reached before the material suffers plastic deformation and during the initial ramp of the loading curve. Thus, the value of the yield stress determined in the test was representative of the material behaviour. However, in the case in which the time needed to reach the equilibrium state, te, was longer than that of the onset of the plastic flow, the test would not provide reliable values for the material yield stress. This time, te, seems to depend only on the specimen length and the elastic wave velocity, $c$, in the material: $t_{e}=n L_{o} / c$, where $n$ is a constant, $\mathrm{L}_{\mathrm{O}}$ the distance between the bar ends (not the gauge length). From our numerical results, $n=5$ is a good and conservative value. However, it is not enough to know te, because it is necessary to ascertain if the material has yielded at that time. The load conditions have been applied to the specimen ends as a relative velocity history with trapezoidal profile. In the rise zone, the velocity, $\mathrm{v}$, can be expressed as: $v=v_{\max } t / t_{r}$, where $v_{\max }$ is the maximum value of $v$ and $t_{r}$ the rise time. Thus, the specimen end has a uniform accelerated motion $\left(a=v_{\max } / t_{r}\right)$. In a similar way, in the plane zone, the motion is uniform with velocity $v=v_{\max }$, and thus the specimen elongation is:
$\Delta \mathrm{L}=\frac{1}{2} a t^{2} \quad\left(\mathrm{t} \leq \mathrm{t}_{\mathrm{r}}\right)$
$\Delta \mathrm{L}=\frac{1}{2} a t_{r^{2}}+v_{\max }\left(t-t_{r}\right)$
$\left(t>t_{r}\right)$ 
On the other hand, the elongation at the yield point, $\Delta \mathrm{L}_{\mathrm{y}}$, is: $\quad \Delta \mathrm{L}_{\mathrm{y}}=\sigma_{\mathrm{y}}$ lo / $\mathrm{E}$, where $\sigma_{\mathrm{y}}, \mathrm{E}$ and $\mathrm{l}_{\mathrm{o}}$ are the yield stress, Young's modulus and the specimen gauge length, respectively. The time corresponding to yielding results:

$$
t_{y}=\sqrt{\frac{2 \sigma_{y} t_{r}}{E \dot{e}_{\max }}} \text { if } t_{y} \leq t_{r} \quad t_{y}=\frac{\sigma_{y}}{E \dot{e}_{\max }}+\frac{1}{2} t_{r} \quad \text { if } t_{y}>t_{r}
$$

where $\dot{e}_{\max }$ is the maximum value of the strain rate during the test. Obviously, yield stress values will be meaningful if $\mathrm{t}_{\mathrm{e}}<\mathrm{ty}$, condition that provides the following criterion to design the specimen:

$\mathrm{l}_{\mathrm{o}}<\frac{\sqrt{2}}{5} \sqrt{\frac{\sigma_{\mathrm{y}} \mathrm{t}_{\mathrm{r}}}{\mathrm{k}^{2} \rho \dot{\mathrm{e}}_{\max }}}$ if $\mathrm{t}_{\mathrm{y}} \leq \mathrm{t}_{\mathrm{r}}$ and $\mathrm{l}_{\mathrm{o}}<\frac{\sigma_{\mathrm{y}}}{5 \mathrm{k} \sqrt{\rho \mathrm{E}} \dot{\mathrm{e}}_{\max }}+\frac{\mathrm{t}_{\mathrm{r}}}{10 \mathrm{k}} \sqrt{\frac{E}{\rho}}$ if $\mathrm{t}_{\mathrm{y}}>\mathrm{t}_{\mathrm{r}}$

where $\mathrm{k}=\mathrm{L}_{0} / \mathrm{l}_{\mathbf{0}}$ is a geometrical parameter. The last criterion guarantees the equilibrium at yielding, considering material effects $\left(\sigma_{y}\right.$ and $\rho$ ), specimen geometry $\left(k\right.$ and $\left.l_{0}\right)$ and experimental set up properties ( $t_{r}$ and $\dot{e}_{\max }$ ). Although, this is a necessary condition, it does not ensure that yielding is produced at a high strain rate. Taking into account the relative velocity profile:

$\dot{\mathrm{e}}=\frac{\mathrm{v}}{\mathrm{l}_{\mathrm{O}}}=\frac{\mathrm{v}_{\max }}{\mathrm{t}_{\mathrm{r}} \mathrm{l}_{\mathrm{o}}} \mathrm{t}=\frac{\dot{\mathrm{e}}_{\max }}{\mathrm{t}_{\mathrm{r}}} \mathrm{t} \quad$ if $\quad \mathrm{t} \leq \mathrm{t}_{\mathrm{r}} \quad$ and $\quad \dot{\mathrm{e}}=\dot{\mathrm{e}}_{\max } \quad$ if $\quad \mathrm{t}>\mathrm{t}_{\mathrm{r}}$

At yielding $\left(t=t_{y}\right): \quad \dot{\mathrm{e}}\left(t_{\mathrm{y}}\right)=\dot{\mathrm{e}}_{\max } \frac{t_{y}}{t_{r}} \quad$ that is $\quad \frac{\dot{\mathrm{e}}\left(t_{\mathrm{y}}\right)}{\dot{\mathrm{e}}_{\max }}=\frac{t_{\mathrm{y}}}{t_{\mathrm{r}}}$

Let us to define a parameter $P$ as: $\quad P=\frac{\dot{e}\left(t_{y}\right)}{\dot{e}_{\max }}$ then $\frac{t_{y}}{t_{r}}=P$

where $\mathrm{P}$ is a parameter for each experimental set up. From the previous expression derived for ty we have:

$$
\mathrm{t}_{\mathbf{r}}=\frac{2 \sigma_{\mathrm{y}}}{\mathrm{E} \dot{\mathrm{e}}_{\mathrm{max}} \mathrm{P}^{2}}
$$

The ideal situation would be $P=1$. Although, experimental difficulties can justify other values for $P$, taking into account that rate sensitivity effects appear with changes in $\dot{e}$ higher than one order of magnitude. (In the experimental results shown in this paper, the yield stress values were measured at strain rates above $500 \mathrm{~s}^{-1}$ ).

Regarding the uniform strain distribution, Nicholas [6], in a previous work, from static experimental data, found a relationship between the material strain field and the relative displacements of the specimen edges. Almost simultaneously, Elwood et al. [7] extended Nicholas' analysis proposing an effective length, obtained from dynamic experimental data, provided by strain gauges attached to the central region of the specimen. Their correction was obtained up to the failure of the strain gauge and it was assumed that this correction does not vary during the test. Additionally, the effective length was considered as independent of the material properties. In our work, a full-numerical modeling of the test has been carried out up to the start of necking demonstrating that the uniform strain distribution hypothesis is not fulfilled during the whole test, which suggests that the results obtained following the usual procedure should be corrected according to the material behaviour regime: a) During the elastic regime the relative displacement between the bar edges is due to the uniform strain of the gauge length plus the deformation of the threaded zone. If only the gauge length is considered when calculating the specimen strain, the material deformation will be overestimated. b) In the plastic regime only the gauge length deform in a uniform way. If the complete gauge length, $1_{0}$, is used to calculate the material strain, the values will be lower than those of the central zone; if only the length of this uniform strain region, $1_{u}$, is 
used, the strain determined will be higher than the actual strain, because the relative displacement of the bars will be totally attributed to the central region. Consequently, it seems logical to average the contribution of each zone, defining an effective specimen length, $l_{e}$, as the mean value of the original gauge length and the length of the uniform strain region:

$$
1_{e}=\frac{1_{0}+l_{u}}{2}
$$

This $l_{e}$ would be the gauge length corresponding to a specimen, which provided the same $\Delta \mathrm{L}$ with a uniform strain distribution. The length $\mathrm{l}_{\mathrm{u}}$ can be determined from the numerical analysis. In the case analyzed in this paper the resulting values were: $l_{0}=13 \mathrm{~mm} ; l_{\mathrm{a}}=5 \mathrm{~mm} ; l_{e}=9 \mathrm{~mm}$. The result of calculating the material strain as the relative displacement of the bars divided by this effective length, and the comparison with the strain in the central region directly measured appear in Figure 6.

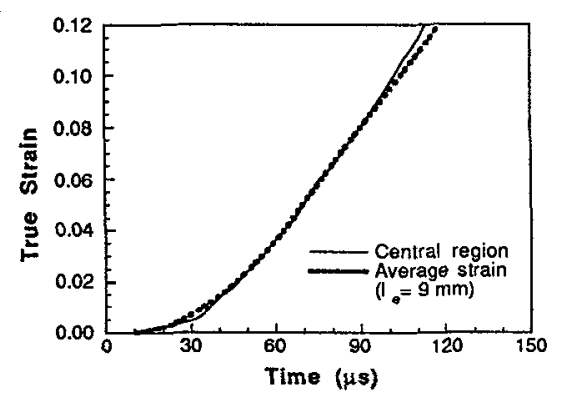

Figure 6. True strain from effective length
In an attempt to extend the previous results, several numerical analyses were carried out varying the geometry and properties of the material tested. The results indicate the following tendencies:

i) Influence of the specimen geometry: When the length/diameter ratio is larger, the effective length is higher. In the range analyzed, the ratio between the radii of the zones near the threads and the specimen diameter has less influence on the effective length.

ii) Influence of the variation of material properties: The effective length decreases when Young's modulus and Yield Stress increases, and the opposite tendency is observed for the variation of the Strain Hardening Slope.

\section{CONCLUSIONS.}

Experimental observations and numerical results have disclosed the actual stress and strain fields of a specimen in a dynamic tensile test in a Hopkinson bar device. The results can be summarized in the following outstanding conclusions:

1) The specimen used in the experiments reaches a quasi-static equilibrium condition clearly before the material initiates plastic flow. Thus, the values of the material yield stress can be obtained from the test. Additionally, some criteria to design properly the test have been derived from a series of numerical analyses, considering material properties, specimen geometry and experimental set up characteristics.

2) The uniform strain hypothesis is not in agreement with experimental and numerical results. Based on numerical analyses, an effective length has been defined which provides a method to calculate the actual material deformation. The influence on this quantity of the specimen geometry and the properties of the material has been estimated.

\section{REFERENCES.}

[1] H. Kolsky . Proc. Royal Soc. B, (1949) Vol 62, 676.

[2] J. Harding, E.D. Wood and J.D. Campbell . J. Mech. Eng. Sci., (1960) Vol 2, pp 88 to 96.

[3] J. Duffy. "Mech. Prop. at High Rates of Strain", (1980) J. Harding Ed., I. of Phy, London.

[4] T. Nicholas. "Dynamic Tensile Testing...", (1980) Technical Report AFWAL-TR-80-4053.

[5] ABAQUS MANUAL (1989), Hibbitt, Karlsonn \& Sorensen Company. USA.

[6] T. Nicholas. Experimental Mechanics, (1981), pp 177 to 185.

[7] S. Ellwood, L.J. Griffiths and D. J. Parry, J. Phys. E., Vol. 15, (1982), 1169 to 1172. 